\title{
Metagenomic Next-generation Sequencing of Cerebrospinal Fluid for the Diagnosis of Central Nervous System infections in HIV-infected patients
}

Nai qing Zheng ( $\sim$ znq1981@qq.com )

Guangzhou Eighth People's Hospital

\section{Pengle Guo}

Guangzhou Eighth People's Hospital

\section{Xiejie Chen}

Guangzhou Eighth People's Hospital

\section{Haolan He}

Guangzhou Eighth People's Hospital

\section{Yueping Li}

Guangzhou Eighth People's Hospital

\section{Linghua Li}

Guangzhou Eighth People's Hospital

\section{Weiping Cai}

Guangzhou Eighth People's Hospital

\section{Research}

Keywords: Metagenomic Next-generation Sequencing, Diagnosis, Central Nervous System infections, HIV

Posted Date: December 27th, 2019

DOI: https://doi.org/10.21203/rs.2.19687/v1

License: (9) (i) This work is licensed under a Creative Commons Attribution 4.0 International License. Read Full License 


\section{Abstract}

Background HIV-infected patients have extremely low immunity and various opportunistic infections. Early diagnosis and treatment of these pathogens is critical for patients with HIV infection, especially those with central nervous system (CNS) infections. Metagenomic next generation sequencing (mNGS) has the advantage of identifying a broad range of pathogens and was suggested as a promising tool in the clinical diagnosis for infectious diseases. The clinical application of mNGS in the diagnosis of CNS infections in patients infected with HIV remains inadequately characterized.Methods We retrospectively analyzed data from 22 patients with suspected central nervous system infections who underwent both mNGS and conventional methods including culture, PCR, X-pert/RIF and antigen testing to explored the utility of mNGS in clinical diagnostic microbiology of CNS infections in HIV-infected patients. Results A total of 22 patients participated in the study between June 2018 and May 2019. The consistency of positive percentage of mNGS compared to clinical diagnosis was significantly higher than that of conventional methods ( $86.36 \%$ vs. $45.21 \%$ ). The proportion of co-infections in mNGS positive samples was significantly higher than that in traditional methods (40.91\% vs. $14.39 \%)$. Sixteen Extra Pathogens in 14 cases identified by metagenomic NGS only, 6 pathogens affected clinical reasoning and 7 pathogens guided antimicrobial therapy.Conclusions MNGS is a powerful diagnostic method for identifying pathogens in central nervous system infections and provide actionable information in some cases. MNGS technology has positive significance for the diagnosis and clinical treatment of central nervous system infection in HIV-infected patients.

\section{Introduction}

HIV infection is a global epidemic[1]. As of 2018, approximately 37.9 million people worldwide were infected with HIV[2]. In 2017, approximately 770,000 people died from diseases caused by HIV infection[2]. The HIV virus attacks human immune cells, causing low immunity. Central nervous system infections are destructive syndromes that remain undiagnosed in most cases [3] . Furthermore, some patient groups, such as those infected with HIV, who often show subtle or non-specific symptoms and signs, are at high risk for encephalitis, accidental infection or unusual pathogens, and are considered to have more serious consequences.[4]. For many patients, the traditional methods requires extensive, often negative, series of tests using a combination of culture, serological, antigen, and molecular methods, leading to delayed or missed diagnoses and is achieved in only $50-80 \%$ of cases $[5,6]$. Failure to diagnose patients with central nervous system diseases in time often results in delayed, inadequate, inappropriate treatment, and increased a high cost burden to the healthcare. This applies in particular to CNS infections, where rapid, accurate identification of pathogens and rapid initiation of antimicrobial treatment are potentially lifesaving.

Metagenomic next generation sequencing ( $\mathrm{mNGS}$ ) is a rapidly evolving molecular technology with great potential to provide a direct, unbiased analysis of the microbial composition of clinical samples without reliance on traditional culture or targeted molecular tests[7, 8]. Cerebrospinal fluid (CSF) is particularly suitable for NGS due to its sterility in healthy individuals. Several recent published reports describing the 
usefulness of mNGS of CSF to identify known and unsuspected pathogens and to discover new microorganisms[9-11]. However, there have been no reports of studies on cerebrospinal fluid (CSF) samples from HIV-infected individuals. CNS infection is more common in patients with severe immunosuppression of $\mathrm{CD}^{+} 4^{+} \mathrm{T}$ cell counts $<200 / \mathrm{ul}$ than in immunocompetent hosts. In immunosuppressed individuals, opportunistic infections (Ols) is the most probably diagnostic considerations and multiple etiologies can coexist.[12].An in-depth understanding of the various etiologies of CNS disease in HIV-infected patients is important for proper assessment and management . Recently, we explored the application of MNGS of CSF in the diagnosis of CNS infections in patients with severe immunosuppression.

\section{Methods}

\section{Study Patients}

Twenty-two HIV-infected patient with a diagnosis of encephalitis or meningitis were recruited from Infectious Diseases Center of Guangzhou Eighth People's Hospital between June 2018 and May 2019. Criteria for diagnosis of encephalitis/meningitis are based on relevant guidelines and/or consensus [13, 14]. Cerebrospinal fluid specimens were tested by clinical microbiology and mNGS respectively. All samples had complete clinical microbiological data. The study was conducted in accordance with the Helsinki declaration and approved by the local institutional review committee. Written informed consent was obtained from All participants.

\section{mNGS of CSF}

\section{DNA Extraction, library construction and sequencing}

0.5-3mL CSF sample from patient was collected according to standard procedures. $1.5 \mathrm{ml}$ microcentrifuge tube with $0.5 \mathrm{ml}$ sample, $1 \mathrm{~g} 0.5 \mathrm{~mm}$ glass bead were placed on a vortex mixer on a horizontal platform and stirred violently for $30 \mathrm{~min}$ at 2,800-3,200 rpm. The $0.3 \mathrm{~mL}$ sample was isolated into a new $1.5 \mathrm{~mL}$ microcentrifuge tube and DNA was extracted using TIANamp Micro DNA Kit (DP316, TIANGEN BIOTECH) according to the manufacturer's recommendations.[15].

DNA libraries were constructed by DNA fragmentation, end-repair, splicing and PCR amplification. Agilent 2100 was used to control the quality of DNA libraries. The qualified libraries were sorted by using the BGISEQ-50 platform[16].

High-quality sequencing data wre obtained by subtracting the low quality sequencing data [17], and then the Burrows-Wheeler Alignment method was used to compute the subtraction of human host sequences mapped to the human reference genome (hg19)[16]. By removing the low complexity reading data and comparing the four microbial genome databases of virus, bacteria, fungi and parasites, the remaining data were classified. The databases were downloaded from NCBI (ftp://ftp.ncbi.nlm.nih.gov/genomes/). It contains 4152 genome-wide sequences of viral taxa, 3446 bacterial genomes or scaffolds, 206 fungi 
associated with human infections, and 140 parasites associated with human disease. The number of unique alignment reads (SMRN) and SDSMRN was calculated (SDSMRN=SMRN* 20000000/ (Clean Reads number)). Using BED Tools to calculate the coverage ratio and depth of each microorganism[18].

\section{Criteria of Positive mNGS Result}

To reduce the influence of potential contamination, we used the following criteria for positive results of CSF mNGS:

1. 1.Bacteria (excluding mycobacteria) and parasites: mNGS identified a microbe (species level) whose SDSMRN of the $\geq 10$ that ranked among the top 5 for bacteria, virus or parasites than that of any other microorganisms.

2. Fungi : mNGS identified a microorganism (species level) with a score of 5-fold higher for a specific pathogen (SDSMRN) than any other fungus due to its low biomass in DNA extraction [19].

3. Mycobacterium tuberculosis (MTB): the result was considered positive when the species or genus level detected by mNGS at least 1 read owing to the difficulty in DNA extraction and low probability of contamination[20].

Statistical analyses and figures were performed using the SPSS statistical package 17.0 software and EXCEL 1810. Depending on their distribution, all data wre expressed as medians with interquartile ranges (IQRs) or as means \pm standard deviation. $P$ values $<0.05$ were considered significant.

\section{Results}

Patients Characteristics Twenty-two patients were finally included, including 21 males (94.54\%) with a median age was 36 years (IQR: 27-46 years). With 17(77.27\%) CT/MRI positive and only 1 patient (4.55\%) on Antiroviral Therapy (Table 1). The cohort consisted mainly of isolated meningitis (13 patients [59.09\%]) or encephalitis (7 patients [31.81\%]), with $9.09 \%$ presenting with myelitis (2 patients). $31.81 \%$ (7 patients) study patients were severely ill, all of whom were admitted to intensive care unit (ICU). The median $\mathrm{CD}^{+} 4^{+} \mathrm{T}$ cell count 74cells/ul (IQR: 13-214 cells/ul). The median time from admission to CSF sampling for metagenomic NGS was 3 days (IQR:0-14 days). The median white blood cell count in CSF was $9 / \mathrm{mm}^{3}$ (IQR: $4-90 / \mathrm{mm}^{3}$ ). 


\begin{tabular}{|l|l|}
\hline Characteristic & Value \\
\hline Age years median(range) & $36 \square 27-46 \% \square$ \\
\hline Male sex - no. (\%) & $21 \square 95.45 \% \square$ \\
\hline Syndrome - no. (\%) & $13 \square 59.09 \% \square$ \\
\hline Meningitis only & $7 \square 31.81 \% \square$ \\
\hline Encephalitis with or without meningitis & $2 \square 9.09 \% \square$ \\
\hline Myelitis with or without meningitis & $15 \square 68.19 \% \square$ \\
\hline Department & $7 \square 31.81 \% \square$ \\
\hline Infectious disease department & $17 \square 77.27 \% \square$ \\
\hline Intensive care unit & $1 \square 9 \% \square$ \\
\hline MRI/CT $+\square$ & $74(13-214)$ \\
\hline Antiroviral Therapy (\%) & $9 \square 4-90 \square$ \\
\hline CD3+4+ T cell count (cells/ul) median(range) & $3 \square 0-14 \square$ \\
\hline White blood cell count in CSF (10E6/L) & 22 \\
\hline Median time after hospital admission that CSF sampling for metagenomic NGS (range) - days \\
\hline All cases & \\
\hline
\end{tabular}

\section{Performance of mNGS for diagnosing CNS infections}

Diagnostic performance of mNGS Relative to Conventional Methods

Twenty-two CSF samples were tested by mNGS and conventional microbiological studies. 19 infections were identified by mNGS, while 13 infections were detected by traditional direct-detection tests alone (defined as culture, PCR, $x$-pert /RIF or CSF antigen tests, excluding serological tests or samples other than CSF). As expected, mNGS increased the sensitivity rate by approximately $40 \%$ in comparison with that of conventional methods ( $86.36 \%$ vs. $45.21 \%$ ) (Table 2 ).

In total, NGS detected 5 bacterial species, of which M. tuberculosis (2cases) was the most common, 20 viral species, of which (EBV)(9) and Human betaherpes virus 5(CMV)(4) were the most common, 6 fungal species, both of which were Cryptococcus (2)and Talaromyces marneffei (2), and 1 parasitic species, of which was the Toxoplasma gondii. (Figure 1-A) Nine co-infections were detected, of which Human gammaherpesvirus 4 (EBV) (7cases) and cytomegalovirus(3cases) was the most common (Figure 1-B). The percentage of co-infection in mNGS positive specimens was notably higher than that of conventional methods positive specimens. ( $40.91 \%$ vs. $14.39 \%)$.

Table 2 Diagnostic performance of mNGS Relative to Conventional Methods 


\begin{tabular}{|c|c|c|}
\hline & mNGS & Conventional methods \\
\hline Positive percent (n/N) (\%) & $86.36 \square 19 / 22 \square$ & $59.09 \square 13 / 22 \square$ \\
\hline \multicolumn{3}{|l|}{ Pathogens } \\
\hline Bacteria $\square \mathrm{n} \square$ & 5 & 2 \\
\hline DNA Viruses $\square \mathrm{n} \square$ & 20 & 12 \\
\hline Fungi $\square n \square$ & 6 & 3 \\
\hline Parasites $\square n \square$ & 1 & 0 \\
\hline Co-infection(n/N) (\%) & $40.91 \square 9 / 22 \square$ & $17.39 \square 4 / 22 \square$ \\
\hline EBV+ Acinetobacter bau-mannii & 1 & 0 \\
\hline EBV+Talaromyces marneffei & 1 & 1 \\
\hline EBV + Mycobacterium tuberculosis & 1 & 1 \\
\hline $\mathrm{EBV}+\mathrm{CMV}+\mathrm{JC}$ polyomavirus & 1 & 0 \\
\hline EBV+Human alphaherpesvirus 3(VZV) & 1 & 0 \\
\hline $\mathrm{CEB}+\mathrm{CMV}+$ Mycobacterium tuberculosis & 1 & 1 \\
\hline CMV+Talaromyces marneffei & 1 & 0 \\
\hline Cryptococcus neoformans+JC polyomavirus & 1 & 0 \\
\hline $\mathrm{EB}+\mathrm{CMV}+$ Aspergillus fumigatus & 1 & 0 \\
\hline EB+CMV+ Talaromyces marneffei & 0 & 1 \\
\hline
\end{tabular}

\section{Concordance Between mNGS and conventional methods in Detection of Pathogens}

In our results, 13 of the 22 cases (59.9\%) with mNGS and traditional methods were both positive, and 2 of the 22 cases $(0.09 \%)$ were both negative. Only mNGS positive was found in 5 cases $(22.73 \%)$, but no positive was found in conventional methods only.For the double positive samples, 6 of the 13 cases were a perfect match, and 2 cases were a complete mismatch. "Partly matched" were found in 5 cases, indicating that at least 1 overlap of pathogens when multiple microbial results were observed (Figure 2).

\section{The clinical implications of mNGStesting in real-world settings.}

Clinician Feedback (Pathogens identified by mNGS in 19 cases)

A total of 27 pathogens were identified in 19 patients (86.36\%). Based on mNGS diagnosis, $4(21 \%)$ ) modified the initial diagnosis, while up to $10(53 \%)$ identified microorganisms corroborated the clinical diagnosis. 2 (10\%) pathogens were not well related with clinical diseases, while $3(16 \%)$ were inconsistent with clinical diagnosis (Figure 3). 
For mNGS identified pathogens, the initial empirical diagnosis was often confirmed by mNGS $(10.53 \%)$ or modified ( $21 \%$ ), while only $16 \%$ were considered unreliable (unsupported) and $10 \%$ uncertain.

\section{Clinical Effect}

The mNGS detected pathogens but not detected by conventional methods included: 3 bacterial species, 4 viral species, 3 fungal species, and 1 parasitic species. Among the pathogens identified by the metagenomic NGS alone, 6 pathogens the clinician declared that the results of mNGS approvingly influenced their clinical reasoning and 7 pathogens guided antimicrobial therapy (Table 3 ).

Table 3 Clinical Effect (16 Extra Pathogens in 14 cases identified by mNGS only)

\begin{tabular}{|c|c|c|c|c|}
\hline & All & $\begin{array}{c}\text { Active } \\
\text { antimicrobial } \\
\text { therapy }\end{array}$ & $\begin{array}{c}\text { Non-active } \\
\text { antimicrobial } \\
\text { therapy }\end{array}$ & $\begin{array}{c}\text { No } \\
\text { antimicrobial } \\
\text { therapy }\end{array}$ \\
\hline \multicolumn{5}{|l|}{$\begin{array}{l}\text { Extra Pathogens identified by } \\
\text { mNGS (n) }\end{array}$} \\
\hline \multicolumn{5}{|l|}{ Bacteria } \\
\hline Acinetobacter baumanniic & 1 & & & 1 \\
\hline Haemophilus parahaemolyticus & 1 & & 1 & \\
\hline Pseudomonas aeruginosa & 1 & & 1 & \\
\hline \multicolumn{5}{|l|}{ DNA Virus $\square n \square$} \\
\hline Human alphaherpes virus 3(VZV) & 2 & 1 & & 1 \\
\hline Human gammaherpes virus $4(\mathrm{EBV})$ & 2 & 1 & & 1 \\
\hline Human betaherpes virus 5(CMV) & 2 & 1 & & 1 \\
\hline JC polyomavirus & 2 & & & 2 \\
\hline \multicolumn{5}{|l|}{ Fungi $\square n \square$} \\
\hline Cryptococcus neoformans & 2 & & & 1 \\
\hline Aspergillus sydowii & 1 & 1 & & \\
\hline Aspergillus fumigatus & 1 & 1 & & \\
\hline \multicolumn{5}{|l|}{ Parasites $\square \mathrm{n} \square$} \\
\hline Toxoplasma gondii & 1 & 1 & & \\
\hline total & 16 & 6 & 2 & 7 \\
\hline
\end{tabular}

\section{Discussion}

We assessed the real-life performance of mNGS in the diagnosis of CNS infections in HIV-infected patients with meningitis, encephalitis, or myelitis, and performed routine microbiological testing in 
parallel. Our results suggest that mNGS identified more potential pathogens in CSF than conventional methods (32 vs. 27). A total of 16 extra pathogens were identified by metagenomic NGS alone. It is worth noting that in 6 of these cases, the clinician adjusted the treatment of 7 pathogens. These results suggest that mNGS can identify unsuspected pathogens that clinicians may ignore due to atypical clinical manifestations and illustrate the prospective clinical value of mNGS detection in CNS infections.

Recently, the problem of mixed infection in immunocompromised hosts has received increasing attention from physicians[21, 22] .Mixed opportunistic infections of the CNS may occur in patients with HIV infection who are not under medical supervision or do not know their HIV status. However, in the present study, it was difficult to diagnose the cooccurrence of two or three common opportunistic infections in immunodeficient patients with $\mathrm{CD}^{+} 4^{+} \mathrm{T}$ cell count $\mathrm{t}<200 / \mathrm{ul}$ by conventional microbiological testing (4/22). Whereas, mNGS demonstrated its significant superiority in terms of opportunistic pathogenic microorganisms in immunosuppressed patients s, nine cases of bacterial, fungal or viral co-infection were identified by mNGS. Furthermore, these immunosuppressed patients are most susceptible to infection by noval and unexpected organisms, while, routine microbiologic testing for suspected infectious are based primarily on previous knowledge of the possible pathogen and are mainly limited to polymerase-chain-reaction (PCR) assay or serology. As demonstrated in our studies, 16 extra pathogens was identified by metagenomic mNGS only, many cases of neurological infections have been unexpectedly diagnosed[23, 10,24]. For these reasons, metagenomics ought to be considered earlier in immunocompromised patients.

Nevertheless, challenges remain, such as contamination or background microbial genes being detected in samples, as reported by other studies[25-27] . Due to the high sensitivity ,contaminations may derived from the following sources: some of which is those with no previous report, and other pathogens that exist in the environment, skin flora or the reagents[28]. The most common bacteria detected by mNGS were Propionibacterium, followed by Cutibacterium and Acidovorax. Therefore, clinicians should carefully examine the patient's medical history, route of infection and source of infection when interpreting mNGS results independently.

There were a few limitations in this research. Firstly, RNA viruses could not be detected by mNGS due to Reverse transcription was not performed to construct a DNA library. Next-generation RNA sequencing should be performed in future studies. Secondly, the number of patients enrolled was relatively small. Further prospective clinical trials with more samples would be warranted to assess the diagnostic performance of mNGS in real-life clinical practice, especially in CNS infections of HIV-infected patients.

\section{Conclusion}

In conclusion, this study demonstrated mNGS has great potential value in the diagnosis of CNS infection, but there is still no clear standard at present, so it is necessary to evaluate it through classical pathogenic methods or clinical treatment effect. Therefore, it is necessary to improve the verification and 
interpretation of the results while optimizing the detection method of mNGS in the clinical diagnosis of neuroinfectious diseases.

\section{Abbreviations}

CNS: Central Nervous System; MNGS: Metagenomic next generation sequencing; PCR: polymerase chain reaction; SPSS: Statistical Pack-age for Social Sciences;; MTB: Mycobacterium tuberculosis; CSF:

Cerebrospinal Fluid; VZV: Human alphaherpesvirus 3

\section{Declarations}

\section{Acknowledgements}

Thanks are given to the patients enrolled in this study.

\section{Authors' contributions}

Conception and design of study: LH-L, WP-C. Data collection: HL-H, YP L. Technical assistance区XJ-C. Analysis and/or interpretation of data: PL-G, Drafting the manuscript: NQ-Z, PL-G. All authors read and approved the final manuscript.

\section{Funding}

This project was funded by Chinese 13th Five-Year National Science and technology major project (no. 2018ZX10302103-002, no. 2017ZX10202102-003-004, no.2018ZX10302104-001-007), the Jointinnovation Program in Healthcare for Special Scientific Research Projects of Guangzhou (no. 201803040002).

\section{Availability of data and materials}

The datasets used and/or analysed during this study are available from the corresponding author on reasonable request

\section{Ethics approval and consent to participate}

The study was conducted in accordance with the Helsinki declaration and approved by the Ethics committee of Guangzhou Eighth People's Hospital. Written informed consent was obtained from All participants.

\section{Consent for publication}

Not applicable.

\section{Competing interests}


The authors declare that they have no competing interests.

\section{References}

1. Cohen MS, Hellmann N, Levy JA, DeCock K, Lange J. The spread, treatment, and prevention of HIV-1: evolution of a global pandemic. J Clin Invest. 2008;118(4):1244-54. doi:10.1172/JCl34706.

2. 2019-UNAIDS-data_en.pdf.https://www.unaids.org/sites/default/files/media_asset/2019-UNAIDSdata_en.pdf. (accessed 14 December 2019)

3. Glaser CA, Honarmand S, Anderson LJ, Schnurr DP, Forghani B, Cossen CK et al. Beyond viruses: clinical profiles and etiologies associated with encephalitis. Clin Infect Dis. 2006;43(12):1565-77. doi:10.1086/509330.

4. Granerod J, Ambrose HE, Davies NW, Clewley JP, Walsh AL, Morgan D et al. Causes of encephalitis and differences in their clinical presentations in England: a multicentre, population-based prospective study. Lancet Infect Dis. 2010;10(12):835-44. doi:10.1016/S1473-3099(10)70222-X.

5. Hasbun R, Rosenthal N, Balada-Llasat JM, Chung J, Duff S, Bozzette S et al. Epidemiology of Meningitis and Encephalitis in the United States, 2011-2014. Clin Infect Dis. 2017;65(3):359-63. doi:10.1093/cid/cix319.

6. Khatib U, van de Beek D, Lees JA, Brouwer MC. Adults with suspected central nervous system infection: A prospective study of diagnostic accuracy. J Infect. 2017;74(1):1-9. doi:10.1016/j.jinf.2016.09.007.

7. Forbes JD, Knox NC, Ronholm J, Pagotto F, Reimer A. Metagenomics: The Next Culture-Independent Game Changer. Front Microbiol. 2017;8:1069. doi:10.3389/fmicb.2017.01069.

8. Gu W, Miller S, Chiu CY. Clinical Metagenomic Next-Generation Sequencing for Pathogen Detection. Annu Rev Pathol. 2019;14:319-38. doi:10.1146/annurev-pathmechdis-012418-012751.

9. Fan S, Ren H, Wei Y, Mao C, Ma Z, Zhang L et al. Next-generation sequencing of the cerebrospinal fluid in the diagnosis of neurobrucellosis. Int J Infect Dis. 2018;67:20-4. doi:10.1016/j.ijid.2017.11.028.

10. Mongkolrattanothai K, Naccache SN, Bender JM, Samayoa E, Pham E, Yu G et al. Neurobrucellosis: Unexpected Answer From Metagenomic Next-Generation Sequencing. J Pediatric Infect Dis Soc. 2017;6(4):393-8. doi:10.1093/jpids/piw066.

11. Simner PJ, Miller S, Carroll KC. Understanding the Promises and Hurdles of Metagenomic NextGeneration Sequencing as a Diagnostic Tool for Infectious Diseases. Clin Infect Dis. 2018;66(5):77888. doi:10.1093/cid/cix881.

12. Kranick SM, Goncalves PH, Stetler-Stevenson M, Aleman K, Polizzotto MN, Little RF et al. Paradoxical central nervous system immune reconstitution syndrome in acquired immunodeficiency syndromerelated primary central nervous system lymphoma. Haematologica. 2015;100(1):e21-4. doi:10.3324/haematol.2014.114736. 
13. Venkatesan A, Tunkel AR, Bloch KC, Lauring AS, Sejvar J, Bitnun A et al. Case definitions, diagnostic algorithms, and priorities in encephalitis: consensus statement of the international encephalitis consortium. Clin Infect Dis. 2013;57(8):1114-28. doi:10.1093/cid/cit458.

14. Solomon T, Michael BD, Smith PE, Sanderson F, Davies NW, Hart IJ et al. Management of suspected viral encephalitis in adults-Association of British Neurologists and British Infection Association National Guidelines. J Infect. 2012;64(4):347-73. doi:10.1016/j.jinf.2011.11.014.

15. Jeon YJ, Zhou Y, Li Y, Guo Q, Chen J, Quan S et al. The feasibility study of non-invasive fetal trisomy 18 and 21 detection with semiconductor sequencing platform. PLoS One. 2014;9(10):e110240. doi:10.1371/journal.pone.0110240.

16. Li H, Durbin R. Fast and accurate short read alignment with Burrows-Wheeler transform. Bioinformatics. 2009;25(14):1754-60. doi:10.1093/bioinformatics/btp324.

17. Schmieder R, Edwards R. Quality control and preprocessing of metagenomic datasets. Bioinformatics. 2011;27(6):863-4. doi:10.1093/bioinformatics/btr026.

18. Quinlan AR, Hall IM. BEDTools: a flexible suite of utilities for comparing genomic features. Bioinformatics. 2010;26(6):841-2. doi:10.1093/bioinformatics/btq033.

19. Schlaberg R, Chiu CY, Miller S, Procop GW, Weinstock G, Professional Practice $C$ et al. Validation of Metagenomic Next-Generation Sequencing Tests for Universal Pathogen Detection. Arch Pathol Lab Med. 2017;141(6):776-86. doi:10.5858/arpa.2016-0539-RA.

20. Simner PJ, Johnson JK, Brasso WB, Anderson K, Lonsway DR, Pierce VM et al. Multicenter Evaluation of the Modified Carbapenem Inactivation Method and the Carba NP for Detection of Carbapenemase-Producing Pseudomonas aeruginosa and Acinetobacter baumannii. J Clin Microbiol. 2018;56(1). doi:10.1128/JCM.01369-17.

21. Crotty MP, Meyers S, Hampton N, Bledsoe S, Ritchie DJ, Buller RS et al. Epidemiology, Co-Infections, and Outcomes of Viral Pneumonia in Adults: An Observational Cohort Study. Medicine (Baltimore). 2015;94(50):e2332. doi:10.1097/MD.0000000000002332.

22. Opperman CJ, Bamford C. Co-infection with Streptococcus pneumoniae and Listeria monocytogenes in an immunocompromised patient. S Afr Med J. 2018;108(5):386-8. doi:10.7196/SAMJ.2018.v108i5.12957.

23. Saylor D, Thakur K, Venkatesan A. Acute encephalitis in the immunocompromised individual. Curr Opin Infect Dis. 2015;28(4):330-6. doi:10.1097/QC0.0000000000000175.

24. Wilson MR, O'Donovan BD, Gelfand JM, Sample HA, Chow FC, Betjemann JP et al. Chronic Meningitis Investigated via Metagenomic Next-Generation Sequencing. JAMA Neurol. 2018;75(8):947-55. doi:10.1001/jamaneurol.2018.0463.

25. Strong MJ, Xu G, Morici L, Splinter Bon-Durant S, Baddoo M, Lin Z et al. Microbial contamination in next generation sequencing: implications for sequence-based analysis of clinical samples. PLoS Pathog. 2014;10(11):e1004437. doi:10.1371/journal.ppat.1004437.

26. Lusk RW. Diverse and widespread contamination evident in the unmapped depths of high throughput sequencing data. PLoS One. 2014;9(10):e110808. doi:10.1371/journal.pone.0110808. 
27. Guan H, Shen A, Lv X, Yang X, Ren H, Zhao Y et al. Detection of virus in CSF from the cases with meningoencephalitis by next-generation sequencing. J Neurovirol. 2016;22(2):240-5. doi:10.1007/s13365-015-0390-7.

28. Brown JR, Bharucha T, Breuer J. Encephalitis diagnosis using metagenomics: application of next generation sequencing for undiagnosed cases. J Infect. 2018;76(3):225-40. doi:10.1016/j.jinf.2017.12.014.

\section{Figures}



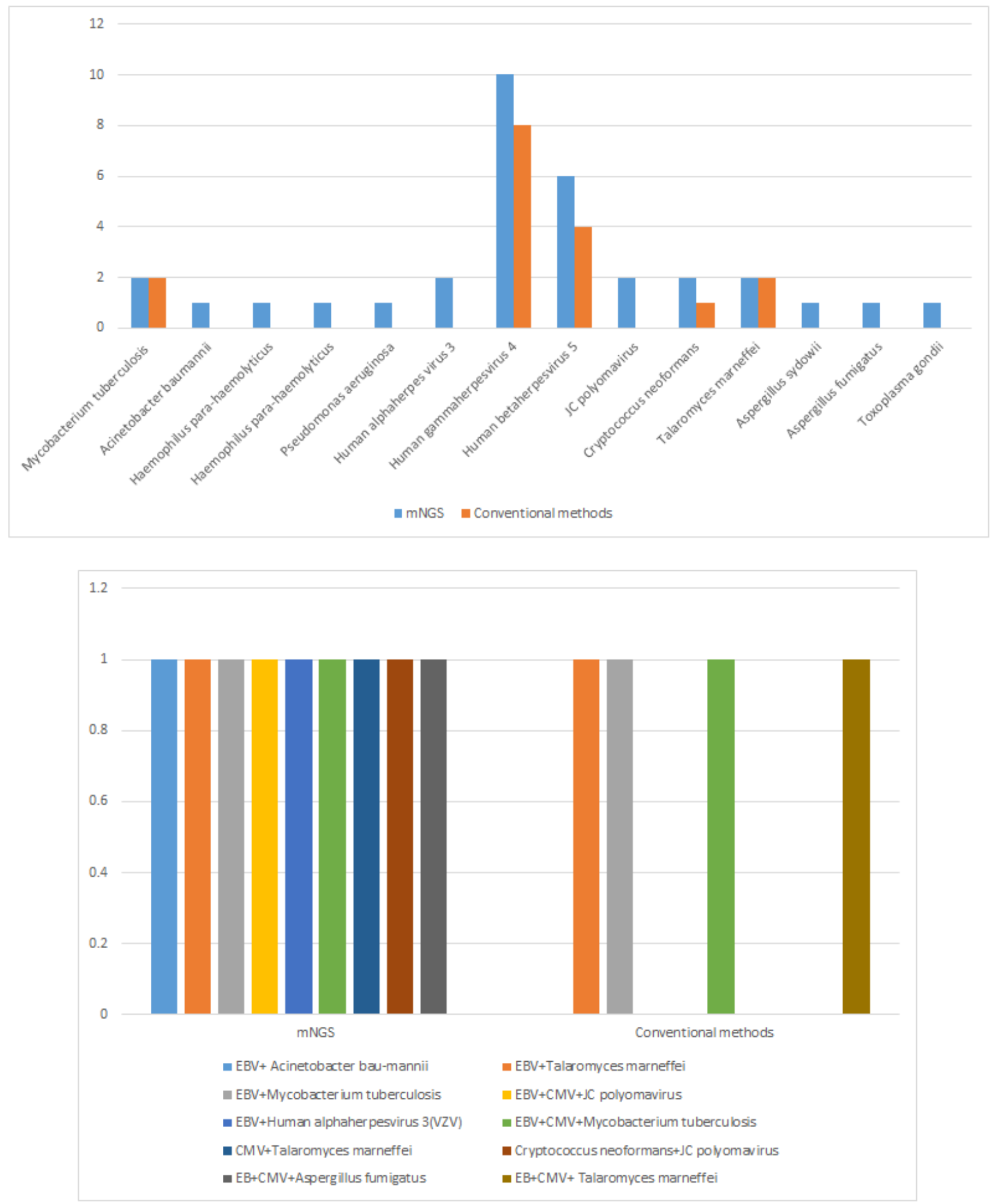

Figure 1

A Comparison Analysis of mNGS and Conventional methods at the Pathogen-type Level. B co-infections 


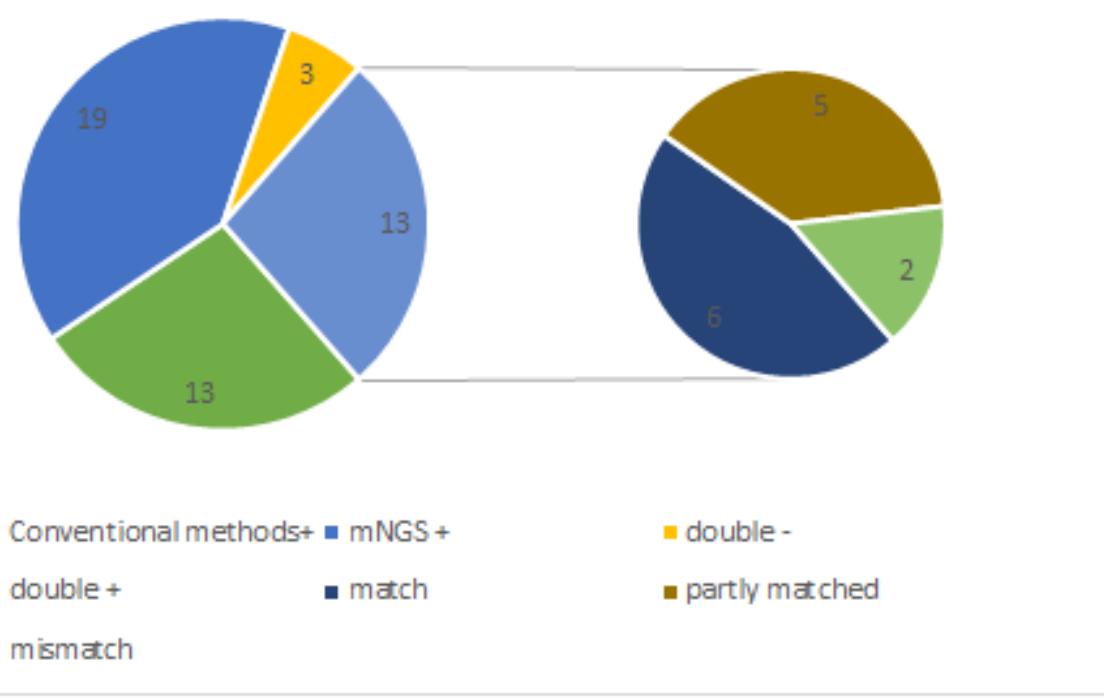

\section{Figure 2}

Concordance Between mNGS and conventional methods for Pathogen Detection

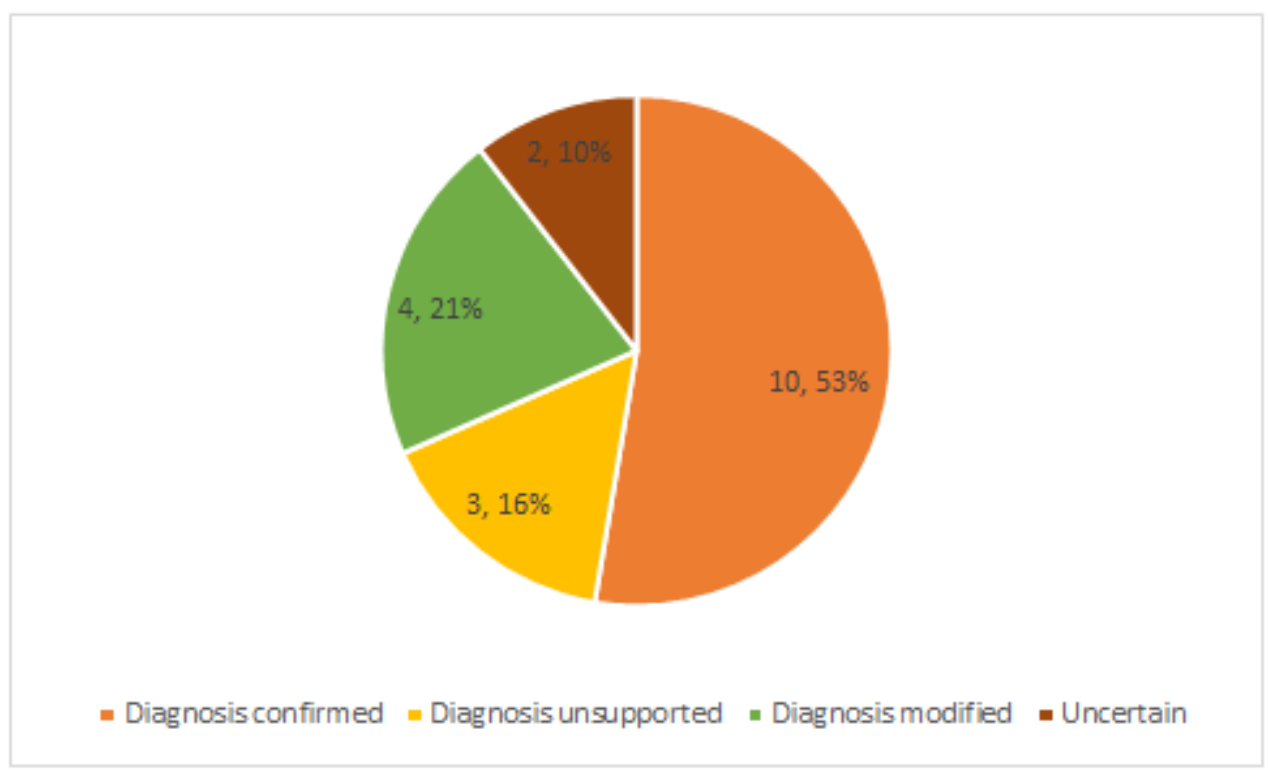

Figure 3

The cocordance of initial diagnosis and mNGS 